\title{
CollisionExplorer: A Tool for Visualizing Droplet Collisions in a Turbulent Flow ${ }^{\star}$
}

\author{
M.V. Rohith ${ }^{1}$, Hossein Parishani ${ }^{2}$, Orlando Ayala ${ }^{2}$, \\ Lian-Ping Wang ${ }^{2}$, and Chandra Kambhamettu ${ }^{1}$ \\ 1 Video/Image Modeling and Synthesis (VIMS) Lab, \\ Department of Computer and Information Sciences \\ http://vims.cis.udel.edu \\ 2 Department of Mechanical Engineering, \\ University of Delaware, Newark, DE, USA
}

\begin{abstract}
Direct numerical simulations(DNS) are producing large quantities of data through their results. Though visualization systems are capable of parallelization and compression to handle this, rendering techniques which automatically illustrate a specific phenomena hidden within larger simulation results are still nascent. In a turbulent flow system, flow properties are volumetric in nature and cannot be displayed in their entirety. Identifying sections of the field data which contain typical and atypical interactions offers a convenient tool to analyze such data. In this paper, we propose methods to explore collision events in DNS studies of droplet collisions in a turbulent flow. Though a variety of geometric models of collisions exist to explain the collision rate, there are few tools available to explore the collisions that really occur in a simulated system. To effectively understand the underlying processes that facilitate collisions, we observe that a global view of all the collisions is required with respect to certain chosen flow parameters together with detailed 3D rendering of the trajectory of a particular collision event. We use GPU based rendering of isosurfaces and droplet trajectories to create such visualizations. The final tool is an interactive visualizer that lets the user rapidly peruse the various collision events in a given simulation and explore the variety of flow characteristics that are associated with it.
\end{abstract}

\section{Introduction}

Direct numerical simulations (DNS) are being used as a quantitative tool for exploring a variety of physical phenomena [6] with advent of high performance computing resources. By encoding elemental rules of interaction of various physical entities, complex and emergent phenomena can be explored. With increasing

\footnotetext{
* This work was supported by the National Science Foundation through grants OCI0904534 and ATM-0730766 and by the National Center for Atmospheric Research (NCAR). NCAR is sponsored by the National Science Foundation. Computing resources are provided by National Center for Atmospheric Research through CISL35751010 , CISL-35751014 and CISL-35751015.
} 
complexity and size of simulations, visualization forms an integral part in the process of scientific discovery [10. Once simulation results are obtained for a particular set of parameters/conditions, scientists use statistics and visualizations of the output to form new hypotheses based on what they observe. The simulations may then be repeated with parameters/conditions which better illustrate the case for or against the hypotheses. Such an approach to discovery is being pursued with a new vigor in the areas of fluid dynamics, particle physics and human behavioral studies. Such studies have resulted in not only a series of exploratory and analytic visualization tools [5]11, but also frameworks which use parallel computing to support visualization of large-scale datasets [13/89].

Visualization frameworks often offer the user a host of options to control the rendering of data including, filtering of data based on multiple attributes, transparency and color controls, viewpoint and field-of-view controls to effectively study a chosen phenomena. These serve to demarcate the region of interest and make the required aspect of the data more perceivable to the human observer. In fact, there has been significant research into the design of non-photorealistic rendering techniques for this purpose. However, if the phenomena to be observed exists in a small spatial and temporal interval, rendering techniques alone may not be able to highlight them effectively. Several query driven approaches [7/5] have been proposed to extract relevant data as defined by the human observer, but these require manual specification of regions of interest. In this paper, we are concerned with observing characteristics of flow that surround collision events between freely suspended droplets in a turbulent flow system.

Given data from a dynamic spatio-temporal simulation with droplets of multiple radii, we attempt to answer the following questions_

* What is the distribution of collision events with respect to flow parameters? * What are the flow structures that surround a given collision event?

There are several works which deal with effect of turbulence on collision through computer simulations. It has been shown that air turbulence can increase the geometric collision kernel by up to $47 \%$, relative to geometric collision by differential sedimentation [1. The collisions are studied to determine the effect of various flow and droplet parameters such as the flow Reynold number, droplet radius, inertia and settling velocities. These help to quantify the collision rates when the flow parameters are changed. However, most of the estimated entities such as the collision kernel are statistical in nature and do not allow for an intuitive mechanistic understanding of individual collision events. In order to better understand the underlying physical processes that lead to collisions, we observe that a global view of all the collisions is required with respect to certain chosen flow parameters together with detailed 3D rendering of the trajectories of a particular colliding droplet pairs. In our tool - CollisionExplorer, we propose to display a global picture of all the collisions with respect to chosen flow attributes such as the local vorticity or the dissipation rate. In this the user can see the distribution of collision events and pick the event that he is interested in studying. Once a particular collision event is selected, we use a combination of $2 \mathrm{D}$ and $3 \mathrm{D}$ rendering techniques to display the features surrounding that 
collision. We show that selective rendering of local structures around an event helps in providing a richer understanding of collision mechanism. We discuss selected related work in Section 2. We describe our approach with results in Section 3 and conclude in Section 4.

\section{Previous Work}

We group our discussion of previous work under three separate subsections. We begin with a review of visualization systems for large-scale data, then briefly comment on some solutions specific to fluid dynamics visualizations.

\subsection{Large-Scale Visualization Frameworks}

Paraview 8], based on Visualization Toolkit (VTK) offers a scalable solution to large-scale data visualization with support for distributed computing resources. Users can create application specific visualizations by using predefined filters or custom scripts. Though it offers several predefined filters and a flexible framework for rendering, some programming is required to create visualizations which illustrate a specific phenomena. VisIt [13] is a scaleable visualization framework which offers analytic tools such as line-out and query. Line-out allows the user to plot the profile of volumetric data along a specified curve, whereas query is a text-driven tool to select regions of data using value interval specifications. These tools are excellent for studying data which vares on structured surface or volume (pressure on a wing, temperature inside an engine), but is of little help when studying dense volumetric data (such as vorticity structures in a turbulent flow). VAPOR [9] is a tool created specifically for studying atmospheric and solar data. Instead of a text-based data selection, it offers a graphical selection of appearance histograms in direct volume visualization. Users can vary color and transparency maps to highlight required sections of data, planar probes can be introduced to better illustrate a localized phenomena. However, the appearance parameters are spatially and temporally uniform limiting the extent to which required region can be highlighted for better perception. The selection of parameters can be done based on the histogram of data values or manually - there is no support for saliency driven parameter selection. Also, since it deals almost exclusively with volumetric data, the system has limited support for particle trajectories and related computations.

\subsection{Fluid Dynamics Visualizations}

Isosurfaces of vorticities, streamlines of fluid velocities and direct volume rendering of velocity/vorticity map are some of the methods commonly used to study turbulent flow structures. These techniques are used to study various turbulence and other flow related phenomena 3]. There are very few visualization works which deal with suspended particles in the fluid. To visualize the interaction of particles with the fluid, the particle positions are superposed on the vorticity/velocity structures. However, most of the visualization plots which capture 
the relation between the particle cluster positions with respect to flow structures are one-dimensional plots. They show the probability of cluster formation with respect to mean vorticity value in a region, or particle settling velocities with respect to flow parameters. Though they quantitatively capture the effect of the interaction, they fail to depict the interaction itself. Since most of these plots are based from statistics that is derived from the simulation output, it is difficult to back-track and identify individual portions of data that illustrate these phenomena. A direct volume rendering of dense data such as vorticity/velocity values is not always convenient to identify specific instances of desired behavior as it is often buried under layers of data which have little relevance. A common method for handling such a scenario is to define a transfer function that maps the data values to colors and transparencies. By controlling the opacities associated with different range of data values, one can see through the irrelevant layers. Though useful for understanding flow structures that arise in various conditions, this method has three drawbacks: (i) the transfer function needs to be manually specified, so it may take several passes to get the parameters right for a particular phenomena, (ii) the transfer function is almost always spatially and temporally uniform limiting the ability to highlight specific regions (iii) the mapping is between data values and colors/transparency which makes it hard to select a transfer function based on interaction between different attributes (e.g., interaction of particle velocity with flow velocity). Hence, we use an adaptive isosurface rendering scheme to render isosurfaces that are relevant to the given interaction. There has been some recent work in visualizing the particle/flow structure interaction with bronchial tubes 12 . The authors provide a visualization showing cross-flow velocities with respect to fluid velocity magnitude inside the tubes. Since their motivation is to study distribution of particle depositions, the methods developed cannot be easily extended to cases of freely suspended particles.

\section{Our Approach}

As noted in Falkovich and Pumir 4], there are two contributions to collisions in turbulent systems - the interaction of the droplets with the local flow shear and the non-local interaction with a distant flow structure due to fluid accelerations and particle inertia. At lower Reynold numbers, the local interaction dominates. In this work, we aim to visualize the local interactions that lead to collision. To obtain an overall picture of the various collisions we introduce a global map which provides the information regarding the flow statistics of colliding droplet pairs in their trajectory. Such a representation helps us study the correlation between the values encountered by the colliding pair. In this overall picture, which we call global map, user can scroll over the various collisions to pick one that has characteristics important to the chosen effect. Once this choice is made, the user can see 2D and 3D maps, which we call region maps, specific to the chosen collision. We use selective isosurface rendering to highlight the structures that may have interacted with the droplets during collision. However, isosurfaces 
provide information about only a single value in the volume. To provide a dense representation of the structure around collision we also create a $2 \mathrm{D}$ map of the surrounding region by choosing the plane which encompasses most of the motion at collision. Before we explain the details of our approach we provide some information about the simulation and the data generated. Results of each method are provided in the corresponding subsections.

\subsection{Simulation Details}

The data used for visualization were obtained by direct numerical simulation of turbulent collisions conducted on a parallel computer (Bluefire) at NCAR. The motion of water droplets of various radii suspended in turbulent air flow was simulated. The flow simulations were performed by solving the Navier-Stokes equations in the spectral domain. The droplet motion is governed by gravity, inertia and drag force. In our simulations, only the droplet behavior was affected by the fluid flow, but the fluid flow was not influenced by droplets. The simulations were conducted on a grid of 256 spatial points in each direction with periodic boundary conditions. Since the dissipative scales of the flow are fully resolved, the physical dimension of each side of the cube depends on the grid resolution. Data generated contained one million droplets which did not interact with each other. The droplets were a bidisperse mixture of 20 and 40 micron $\downarrow$, droplets. The data was obtained via 3000 time steps of simulation sampled at every 15 steps. There were a total of seventeen thousand collisions recorded with approximately 15,500 collisions between a droplet of 40 microns and another of 20 microns, and 1,500 collisions where both the colliding droplets were $40 \mathrm{mi}$ crons. Collisions among 20 micron droplets were fewer than fifty. The turbulence has reached a statistically stationary state by running six eddy turnover times before obtaining flow and droplet data. The details of the simulation can be found in 2 .

\subsection{Global Map}

As noted earlier, the function of the global map is to visualize the overall distribution of a statistics up to the point of collision and also provide a correlation between the values encountered by a particular colliding pair. The data about a collision event contains a number of elements that may be abstracted away for the sake of statistical analysis. Since the simulation volume is considered to be homogeneous, the exact location of a collision is not important. Also, since the characteristics of the flow are nearly time invariant, the exact time step of the collision can be omitted. Instead we concentrate on characteristics of flow at the point of collision, for example, the vorticity and dissipation rate values at the point of collision. Hence we need to plot these flow attributes along droplet trajectory. Since the absolute location of the droplet cannot be used, we use the distance between the colliding pairs as the parameter. Hence if we plot the flow attributes versus the relative distance between the colliding pairs, we ensure a space-time invariant representation. It must be noted that since the boundary 


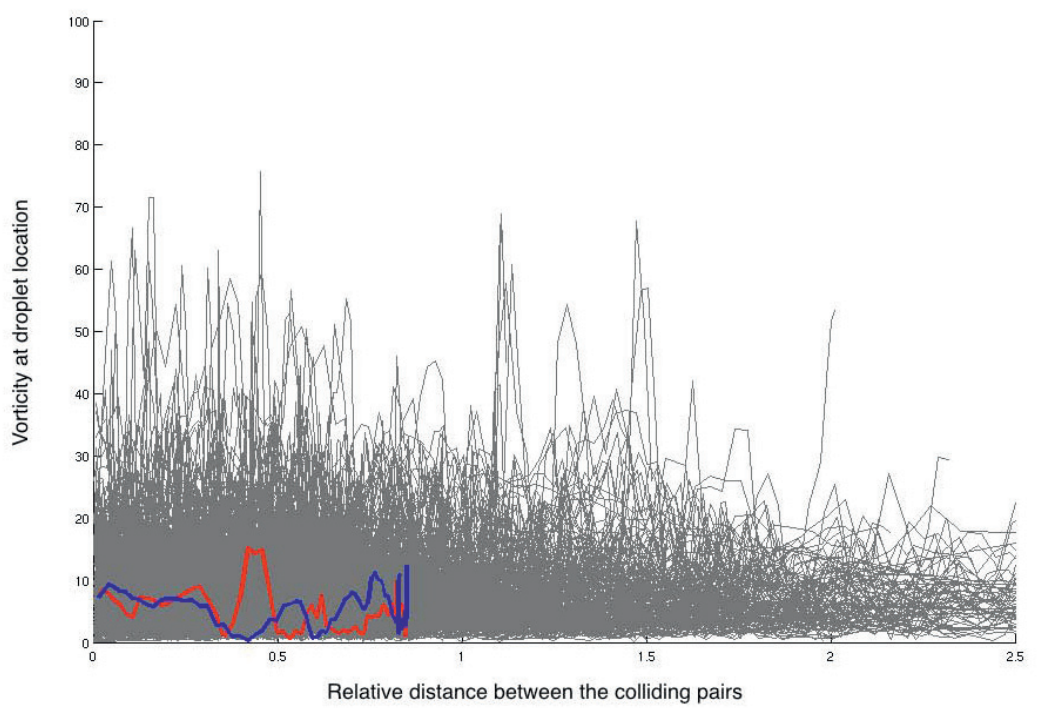

Fig. 1. Global map of collisions based on vorticity values. Horizontal axis is the relative distance between the colliding pair. Vertical axis is the vorticity value at droplet location.

conditions are periodic (a droplet exiting the volume on one face will reappear on the opposite face), we must ensure that the distance between the pair of droplets is measured within a single volume. For this, volume exits and entries are noted for every droplet and part of the trajectory is shifted to ensure that the trajectory is continuous. In the global map, if the horizontal axis were to represent the distance between the pair and the vertical axis represented a flow attribute say vorticity of each droplet in a colliding pair - the pair of droplets would start out away from the vertical axis and then move left. At collision, the distance between them would be zero, hence this point would lie on the vertical axis. The vertical offset of this point will indicate the flow attribute at point of collision. Examples of this are shown in Figure 1 and Figure 2 for vorticity and dissipation rate global maps for collisions between 40-20 micron colliding droplets. It can be seen that an overall distribution of flow attribute now emerges. For example, from Figure 1 we can infer that collisions are less likely in regions containing high vorticity values. The user can then select a pair whose characteristics need to be studied and the region maps for those will be generated as described in the next section.

\subsection{Region Maps}

We generate two kinds of region maps. A 3D map showing the trajectory of droplets in the simulation volume together with isosurfaces. This enables us to visualize the structures which may have interacted with droplet at time of 


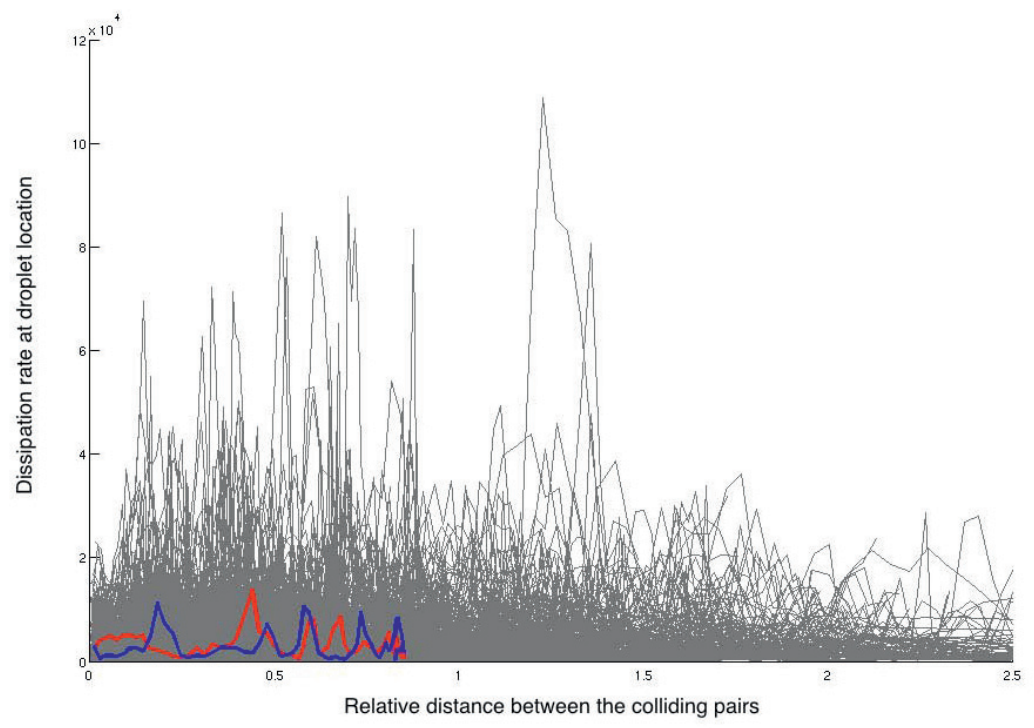

Fig. 2. Global map of collisions based on dissipation rate values. Horizontal axis is the relative distance between the colliding pair. Vertical axis is the dissipation rate at droplet location.

collision. However, since we cannot obtain an idea of the dense field of the flow attribute in this region from isosurface alone, we project the trajectory onto a plane containing the most of the trajectory. The values of required attributes are obtained on this surface using interpolation. When this is visualized it provides us with a dense representation of the region.

Dense 2D region map. To obtain the dense 2D map, we have to decide a plane whose visualization contains the most information regarding the collision event. Consider the pair of droplets as in Figure 3, the droplets are located at positions P1 and P2 with their instantaneous velocities V1 and V2. Since the plane must contain the local trajectory of the droplets, it should preferably contain both V1 and V2. Since the relative position of the droplets must not be distorted, it must contain P1-P2. However, all these constraints cannot be satisfied by a single plane, hence we use an plane which approximately satisfies these constraints. Its formulation may be obtained in closed form as a plane passing through the midpoint between the droplet positions and containing the vectors (V1+V2) and (P1-P2). In cases where this leads to a degenerate solution, the plane from the previous timestep is used. Figure 4 shows the calculated plane for a pair of trajectories. Note that it lies along the trajectories and provides the least distortion for their projections. Figurer 5 shows the slice of vorticity data sampled along that plane. The particle positions are also indicated. 


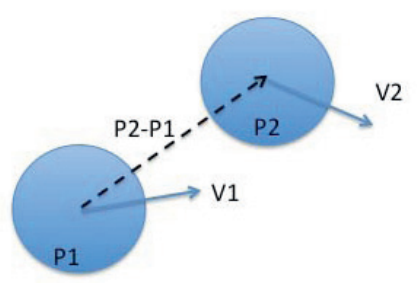

Fig. 3. Two droplets at positions $\mathrm{P} 1$ and $\mathrm{P} 2$ with instantaneous velocities $\mathrm{V} 1$ and $\mathrm{V} 2$

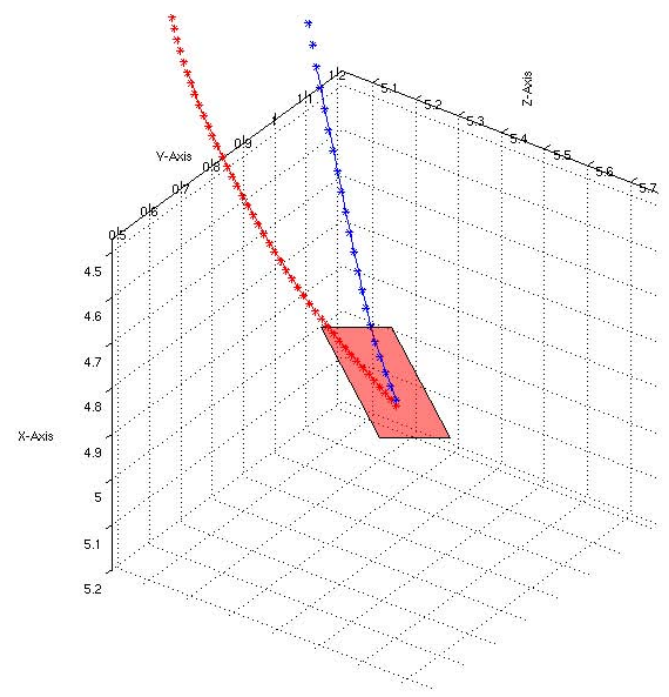

Fig. 4. Droplet trajectories and the plane estimated for projection

Selective isosurface rendering. As seen in Figure [6, rendering isosurfaces together with droplet trajectory does not provide us with a clear method of visualizing the interaction between the droplet and the flow. Hence we propose a method called selective isosurface rendering, in which only those surfaces near a given point of interest are rendered. Since the surfaces must not be truncated or distorted, we cannot just filter the vertices of the isosurface mesh based on distance. We perform connected component analysis on the isosurface mesh to obtain segments of faces which are connected together. Once this is performed, we can choose seed points as those vertices which lie close to the point of interest. Only the segments that contain the seedpoints are rendered. This framework was implemented using Nvidia CUDA library. It was tested on an Intel Pentium Core 2 Duo PC with 3GB RAM and Nvidia GeForce 570 video card with $1.25 \mathrm{~GB}$ memory. The vorticity data and the particle trajectories were obtained from the simulation. The data consisted of vorticity values on a $256 \times 256 \times 256$ grid. The isosurface estimation was carried out using the marching cubes implementation 


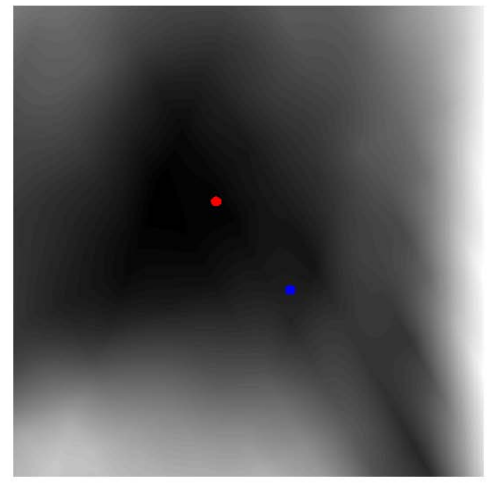

Fig. 5. Droplet positions plotted on the vorticity data sampled from the estimated plane. Darker colors indicate lower vorticity. The red and blue markers correspond to the droplets seen in Fig 4

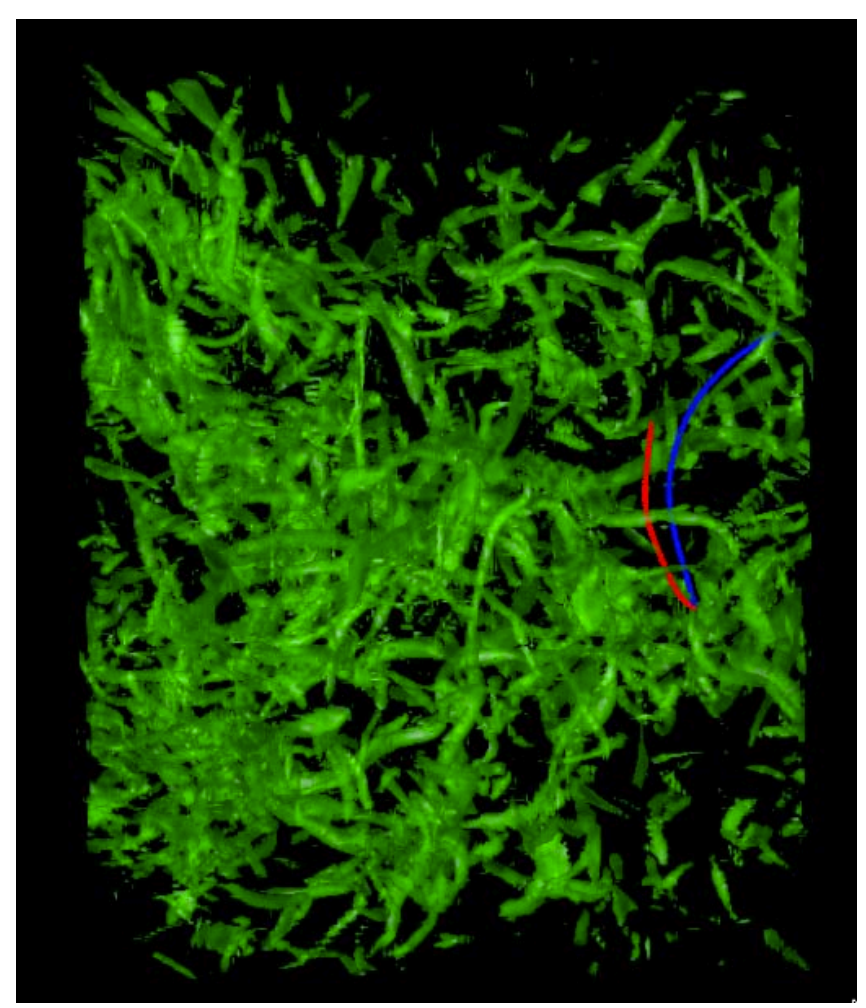

Fig. 6. Isosurface of vorticity (at a value of three times the average vorticity) with droplet trajectories 


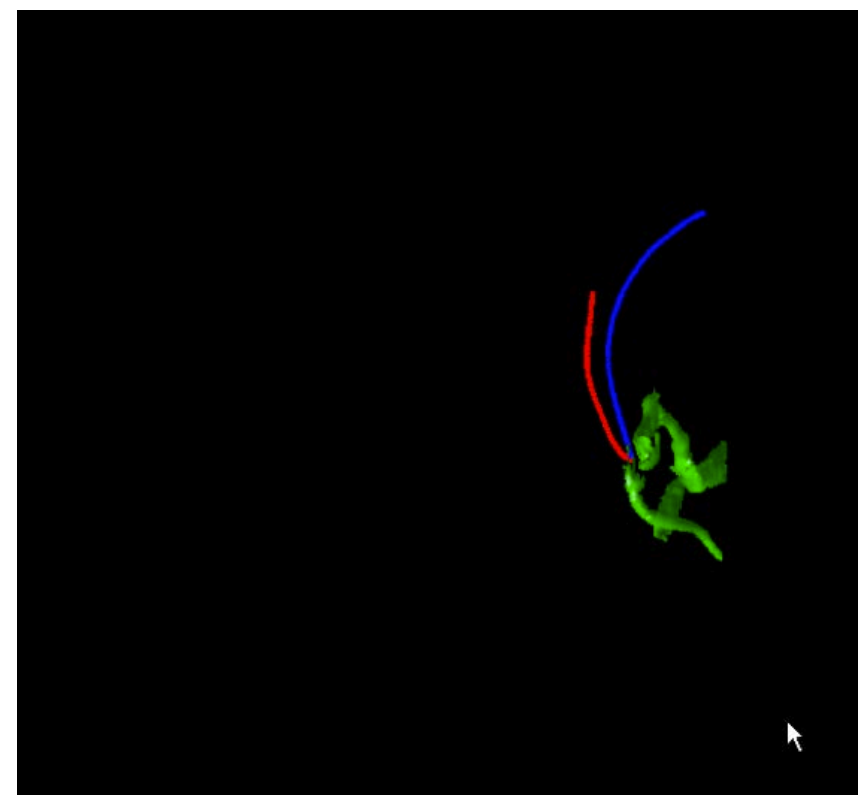

Fig. 7. Selected isosurfaces of vorticity at point of collision (at a value of three times the average vorticity) with droplet trajectories

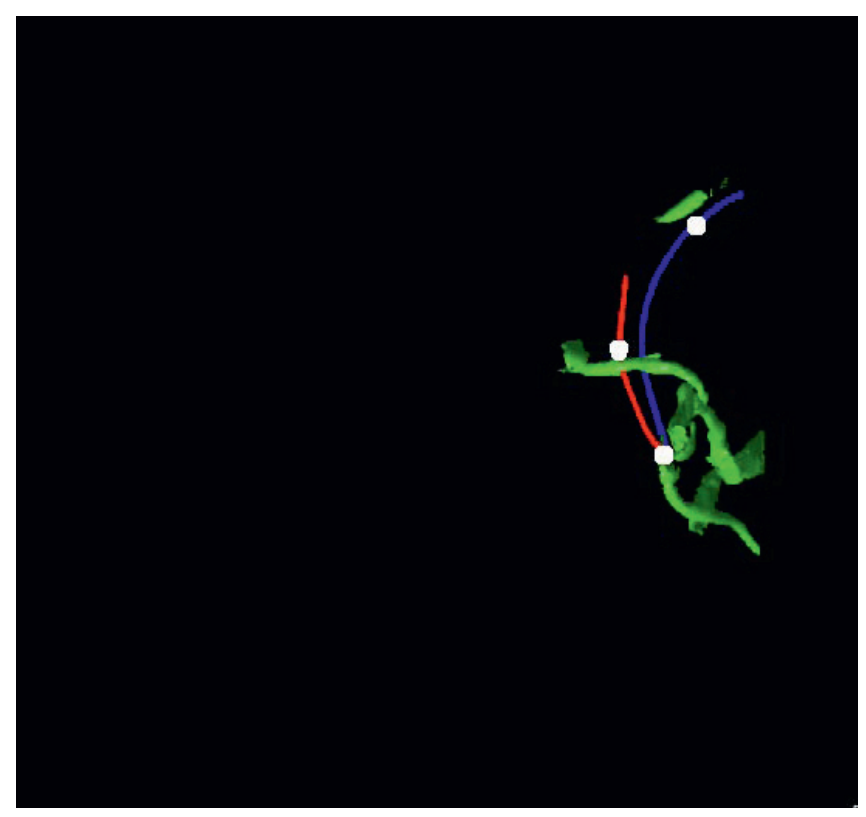

Fig. 8. Time summary of droplet flow structure interaction. The flow structures around three points highlighted in white are shown using isosurfaces from the corresponding timesteps. 
provided in the library. It was suitably modified to include the connected component analysis and segment selection. The algorithm was further optimized by selecting the faces directly using face-walking (traversing adjacent connected faces). The graphics was rendered at a frame rate of $20 \mathrm{fps}$ in $1024 \times 1024$ resolution. Figure 7 shows the result of the same data with selected isosurface rendering. We can extend this method to combine selected isosurfaces at different timesteps to create a time summary of the flow interaction. This is shown in Figure 8

\section{Conclusion}

Visualizing results of large-scale simulation studies grows more challenging with increasing complexity and scale range of experiments. Most visualization systems require extensive user interaction, in-depth domain knowledge and manual scripting to generate illustrations of desired phenomena from the simulation results. Localizing the region of interest in time and space requires manually sifting through the entire dataset which can be laborious and time consuming. Study of turbulent flow systems is an area that has been actively explored using numerical simulations.

In this paper, we report a tool called CollisionExplorer which allows users to interactively explore the various collision events that occur in a simulation. We provide the user with a global map which contains the summary of all the collisions and the user can then choose a specific collision based on his interest. Once a particular collision is chosen, we provide 2D and 3D region maps which displays the sparse and dense representations of flow near the region of collision. Using plane projection and selective isosurface rendering we are able to provide efficient representations of dense volumetric data. In the future, the global map may be made more discriminative with respect to collision mechanisms and more flow attributes may be included in both global and region maps.

\section{References}

1. Ayala, O., Rosa, B., Wang, L.P., Grabowski, W.W.: Effects of turbulence on the geometric collision rate of sedimenting droplets. Part 1. Results from direct numerical simulation. New Journal of Physics 10(7) (July 2008)

2. Ayala, O., Grabowski, W.W., Wang, L.P.: A hybrid approach for simulating turbulent collisions of hydrodynamically-interacting particles. J. Comput. Phys. 225, 51-73 (2007)

3. Clyne, J., Mininni, P., Norton, A., Rast, M.: Interactive desktop analysis of high resolution simulations: application to turbulent plume dynamics and current sheet formation. New J. Phys. 9, 301 (2007)

4. Falkovich, G., Pumir, A.: Sling effect in collisions of water droplets in turbulent clouds. Journal of the Atmospheric Sciences 64(12), 4497-4505 (2007)

5. Gosink, L.J., Anderson, J.C., Bethel, W., Joy, K.I.: Variable interactions in querydriven visualization. IEEE Trans. Vis. Comput. Graph. 13(6), 1400-1407 (2007)

6. Johnson, C.R., Huang, J.: Distribution-driven visualization of volume data. IEEE Trans. Vis. Comput. Graph. 15(5), 734-746 (2009) 
7. Kendall, W., Glatter, M., Huang, J., Peterka, T., Latham, R., Ross, R.: Expressive feature characterization for ultrascale data visualization. Journal of Physics (2010)

8. Law, C.C., Henderson, A., Ahrens, J.: An application architecture for large data visualization: a case study. In: Proceedings of the IEEE 2001 Symposium on Parallel and Large-data Visualization and Graphics, PVG 2001, pp. 125-128. IEEE Press, Piscataway (2001)

9. Lum, E., Ma, K.L., Clyne, J.: A hardware-assisted scalable solution for interactive volume rendering of time-varying data. IEEE Transactions on Visualization and Computer Graphics 8(3), 286-301 (2002)

10. McCormick, P., Anderson, E., Martin, S., Brownlee, C., Inman, J., Maltrud, M., Kim, M., Ahrens, J., Nau, L.: Quantitatively driven visualization and analysis on emerging architectures. Journal of Physics: Conference Series 125(1), 012095 (2008)

11. Roberts, J.C.: Exploratory visualization using bracketing. In: Costabile, M.F. (ed.) AVI, pp. 188-192. ACM Press, New York (2004)

12. Soni, B., Thompson, D., Machiraju, R.: Visualizing particle/flow structure interactions in the small bronchial tubes. IEEE Transactions on Visualization and Computer Graphics 14, 1412-1427 (2008)

13. Weber, G.H., Ahern, S., Bethel, E.W., Borovikov, S., Childs, H.R., Deines, E., Garth, C., Hagen, H., Hamann, B., Joy, K.I., Martin, D., Meredith, J., Prabhat, Pugmire, D., Rübel, O., Van Straalen, B., Wu, K.: Recent advances in visit: Amr streamlines and query-driven visualization. In: Numerical Modeling of Space Plasma Flows: Astronum-2009 (Astronomical Society of the Pacific Conference Series), vol. 429, pp. 329-334 (2010) lBNL-3185E 